\title{
Status and prospects for solar and atmospheric neutrinos
}

TAKAAKI KAJITA* ${ }^{*}$

ICRR and IPMU, Univ. of Tokyo,

Kashiwa-no-ha 5-1-5, Kashiwa, Chiba 277-8582, Japan.

E-mail: kajita@icrr.u-tokyo.ac.jp

Atmospheric and solar neutrino experiments have been contributing substantially to the understanding of the neutrino masses and mixing angles. Recently, several new data have been presented from solar and atmospheric neutrino experiments. These new data are discussed together with the constraints on oscillation parameters. There are still exciting, future solar and atmospheric neutrino studies. The prospects for these experiments are also discussed.

10th International Workshop on Neutrino Factories, Super beams and Beta beams June 30 - July 52008

Valencia, Spain

\footnotetext{
* Speaker.

${ }^{\dagger}$ This work has been supported in part by Grand-in-Aid for Scientific Research of JSPS
} 


\section{Introduction}

In the last 10 years after the discovery of neutrino oscillations [1], there has been much progress in our understanding of neutrino oscillations. Atmospheric and solar neutrino experiments have played essential roles in this period. Small but finite neutrino masses are believed to be related to the physics at the very high energy scale. Furthermore, the physics of the neutrino masses might be related to the baryon asymmetry of the Universe. It is likely that the neutrino physics contributes to our deeper understanding of the nature. Therefore we should try to get as much information as possible from neutrino oscillation experiments. In this paper, status and prospects for solar and atmospheric neutrino experiments are discussed.

In the 3-flavor neutrino oscillation framework, there are 3 mixing angles $\left(\theta_{12}, \theta_{23}, \theta_{13}\right)$, one $\mathrm{CP}$ phase $(\delta)$, and 2 neutrino mass squared differences $\left(\Delta m_{12}^{2}, \Delta m_{23}^{2}\left(=\Delta m_{13}^{2}\right)\right)$. Since $\theta_{13}$ is known to be small and since $\Delta m_{12}^{2}<<\Delta m_{13}^{2}$, it is approximately correct to assume the 2 flavor oscillation approximation. Therefore, in most part of this paper, we interpret the solar and atmospheric neutrino data within the 2 flavor oscillation framework.

\section{Results from solar neutrino experiments - Measurements of $\sin ^{2} \theta_{12}$ and $\Delta m_{12}^{2}$ -}

SNO finished the ${ }^{3} \mathrm{He}$ phase (NCD phase) of the experiment in the late 2006. Data from this phase have been published recently [2]. In the NCD phase, neutrons produced by neutral current (NC) interactions in $\mathrm{D}_{2} \mathrm{O}$ are measured by an array of ${ }^{3} \mathrm{He}$ proportional counters;

$$
n+{ }^{3} \mathrm{He} \rightarrow p+t+0.76 \mathrm{MeV}
$$

Figure 1 shows the NC signal observed in the ${ }^{3} \mathrm{He}$ counters during 385 live days. A signal peak is clearly seen above the background.

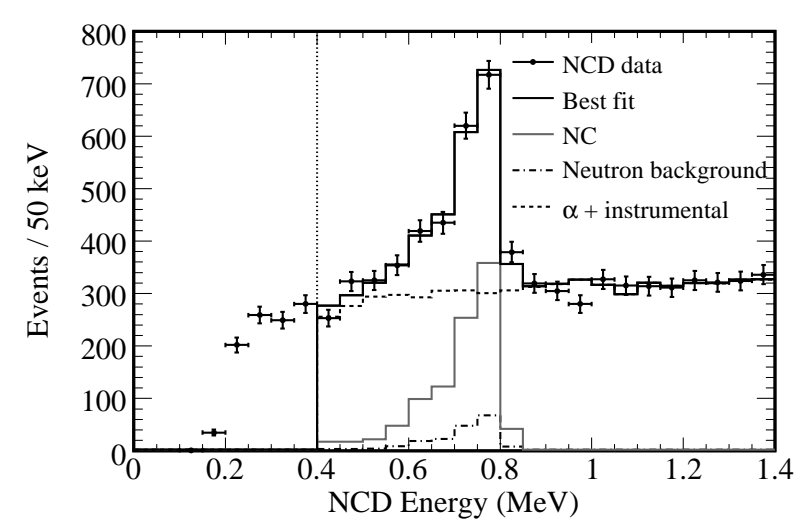

Figure 1: Neutron counter energy spectrum together with Monte Carlo predicted signal and background spectra as well as the best fit to the data. Data from 385 live days of SNO NCD phase are shown [2].

Due to the completely different detection method of the $\mathrm{NC}$ signal, the ${ }^{3} \mathrm{He}$ phase has different systematic errors from the pure $\mathrm{D}_{2} \mathrm{O}$ and salt phases, and therefore gives an important check of the earlier data. A smaller contamination of the NC events in the NCD phase in the measurement of 
charged current (CC) and elastic scattering (ES) interactions by the Cherenkov detection should also be noticed.

The observed NC and CC fluxes and the CC/NC flux ratio in the NCD phase are [2];

$$
\begin{aligned}
& \phi_{N C}=5.54_{-0.31}^{+0.33}(\text { stat })_{-0.34}^{+0.36}(\text { syst }) \\
& \phi_{C C}=1.67_{-0.04}^{+0.05}(\text { stat })_{-0.08}^{+0.07}(\text { syst }) \\
& C C / N C=0.301 \pm 0.033 \text { (total }) .
\end{aligned}
$$

We find that the measured fluxes and the ratio are essentially consistent with the previous measurements [3]. We also notice that the ratio is slightly smaller than the previous value, although the difference is not statistically significant. Since the CC/NC ratio is approximately equal to $\sin ^{2} \theta_{12}$, it will be very interesting to know the best fit $\sin ^{2} \theta_{12}$. We will discuss the fitted oscillation parameters later.

KamLAND is a long baseline reactor neutrino experiment. The mean distance to the surrounding reactors is $180 \mathrm{~km}$. This experiment is contributing to the oscillation studies very substantially. Since there is no other paper in these proceedings that discusses the KamLAND data in detail, we discuss the main result from KamLAND in this paper. The KamLAND collaboration published the results based on 2,881 ton.year detector exposure [4]. KamLAND measures the energy spectrum of candidate $\bar{v}_{e}$ interactions. Then assuming a single distance from the reactors to KamLAND ( $\mathrm{L}_{0}$ $=180 \mathrm{~km}$ ), the oscillation probabilities as a function of L/E are plotted (L/E plot). Figure 2 shows the observed L/E distribution of the candidate reactor neutrino events. A clear L/E dependent sinusoidal oscillation has been observed.

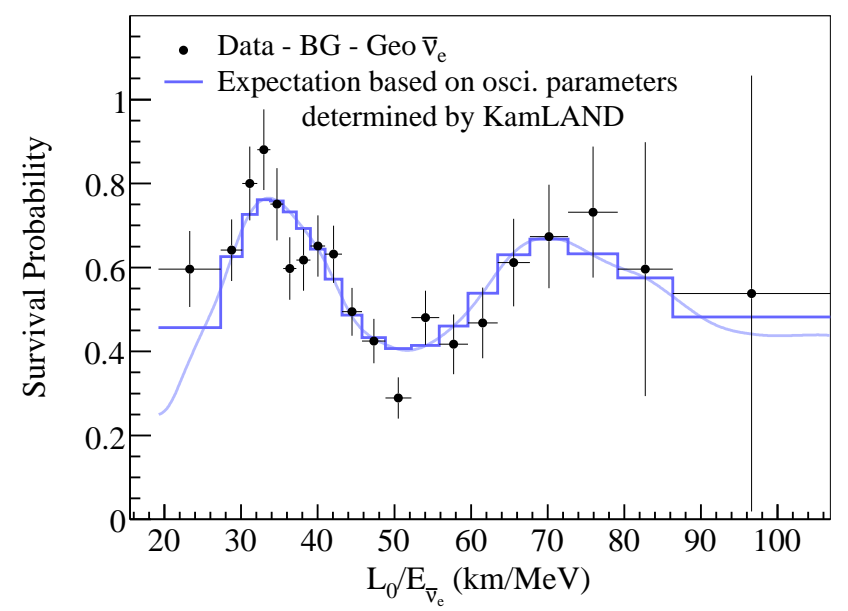

Figure 2: Ratio of the background subtracted reactor $\bar{v}_{e}$ spectrum to the no-oscillation expectation as a function of $\mathrm{L}_{0} / \mathrm{E}$. 2881 ton year of the KamLAND data are used. $\mathrm{L}_{0}=180 \mathrm{~km}$ is assumed. (From Ref. [4].)

Finally, we remark that a substantial fraction of the solar neutrino data related to the oscillation parameter measurements are obtained by the measurements of the high-energy solar neutrinos above $\sim 5 \mathrm{MeV}$. It is important to confirm that the oscillation probability for lower-energy solar neutrino is as expected by the standard LMA solution. In addition, there have been suggestions that new physics effects might appear in the oscillation probability for the sub-MeV solar neutrinos $[5,6]$. Therefore, it is important to study the sub-MeV solar neutrinos. 
BOREXINO is a real-time ${ }^{7} \mathrm{Be}$ solar neutrino experiment. ${ }^{7} \mathrm{Be}$ solar neutrinos are measured by the neutrino-electron scattering. It has been taking data since May 2007 [7]. The updated data were presented this year with the total exposure of 192 live days [8]. The observed event rate of ${ }^{7} \mathrm{Be}$ solar neutrinos was; $49 \pm 3$ (stat) \pm 4 (syst)/counts/day/100tons, while the predicted event rate by the LMA solution was $48 \pm 4 /$ counts/day/100tons. The observed event rate of the ${ }^{7} \mathrm{Be}$ solar neutrinos was consistent with the prediction by the LMA solution, further confirming the standard oscillation scenario.

These data are used to constrain the oscillation parameters [9]; $\sin ^{2} \theta_{12}$ and $\Delta m_{12}^{2}$. Figure 3 shows the allowed oscillation parameter region from the solar and the KamLAND experiments. The $\Delta m_{12}^{2}$ region is most accurately determined by the KamLAND energy spectrum. On the other hand, the mixing parameter is strongly constrained by the SNO CC/NC measurement, because the $\mathrm{CC} / \mathrm{NC}$ flux ratio is approximately equal to $\sin ^{2} \theta_{12}$. We also note that the high statistics KamLAND data begin to constrain the mixing parameter as well. From the global fit [9], $\theta_{12}=33.5_{-1.0}^{+1.3}$ deg. and $\Delta m_{12}^{2}=7.65_{-0.20}^{+0.23} \times 10^{-5} \mathrm{eV}^{2}$ at $1 \sigma$ has been obtained. We find that the parameters are already determined accurately.
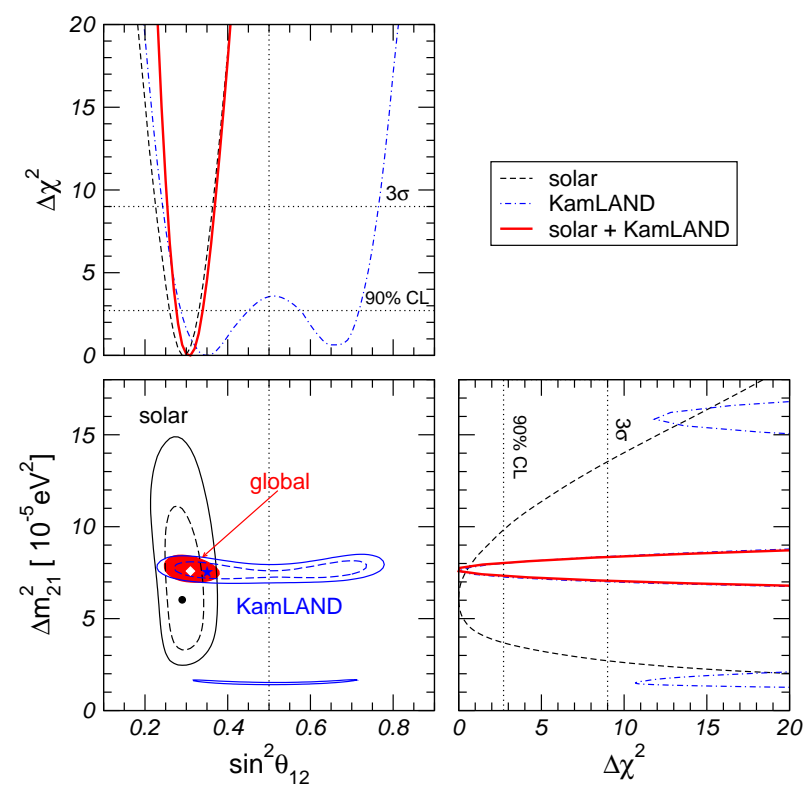

Figure 3: Allowed region of neutrino oscillation parameters by the solar and the KamLAND experiments. (From Ref. [9].)

\section{Results from atmospheric neutrino experiments - Measurements of $\sin ^{2} 2 \theta_{23}$ and $\left|\Delta m_{23}^{2}\right|-$}

The total exposure of the Super-Kamiokande (Super-K) atmospheric neutrino data in the whole Super-K-I [10] and -II periods is $141 \mathrm{kton} \cdot$ years. Recently, the Super-K collaboration updated the atmospheric neutrino analysis with a new flux model [11], Monte Carlo simulation and event reconstruction [12]. As an example, the updated L/E distribution is shown in Fig. 4, and is explained well by the 2-flavor $v_{\mu} \rightarrow v_{\tau}$ oscillations. 


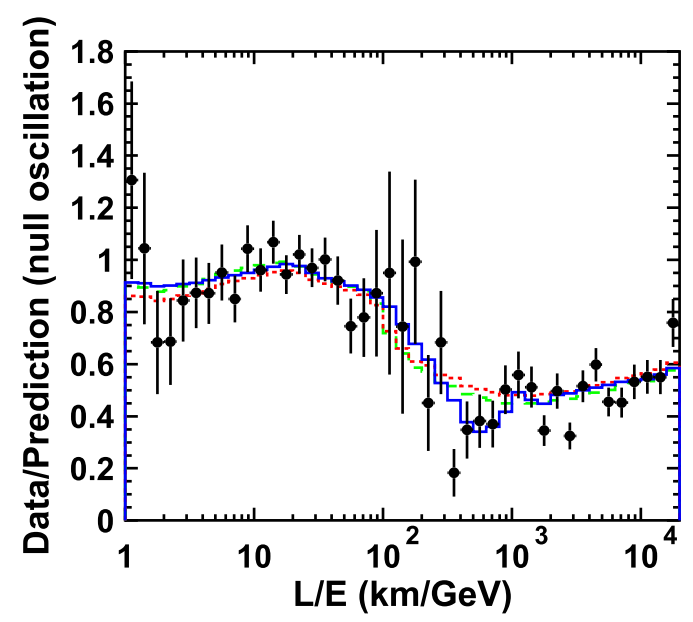

Figure 4: $L / E$ distributions observed in Super-K-I+II (141 kton·year exposure, preliminary). The solid (blue), dashed (green), and dotted (red) histograms show the best-fit predictions based on neutrino oscillation, decay and decoherence models, respectively.

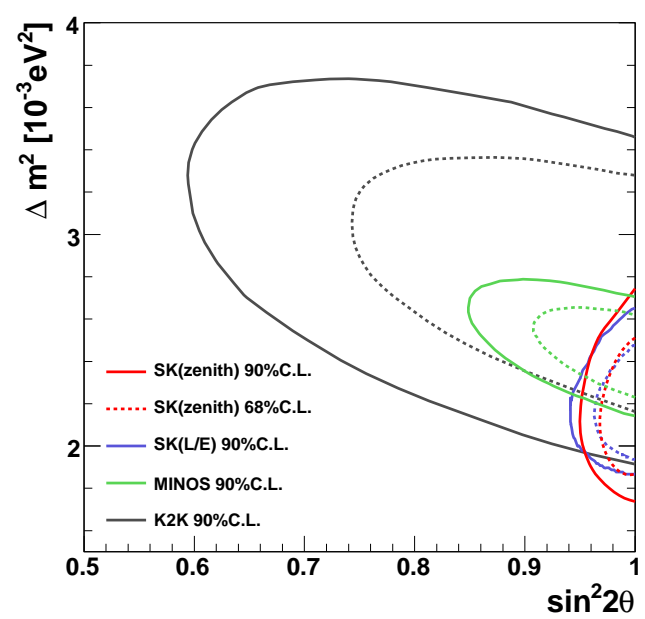

Figure 5: Estimated allowed regions of $v_{\mu} \rightarrow v_{\tau}$ oscillation parameters from the atmospheric [12] and long baseline neutrino oscillation experiments [13], [14] at 68\% C.L. (dotted) and 90\% C.L. (solid).

Figure 5 shows the estimated allowed regions of 2-flavor $v_{\mu} \rightarrow v_{\tau}$ oscillation parameters from the atmospheric and the long baseline neutrino oscillation experiments. We find that the allowed regions overlap well, suggesting further that the standard 2-flavor neutrino oscillation approximation is fine. From this figure, we find that the most accurate information on $\Delta m_{23}^{2}$ is obtained by the long baseline experiments, while the mixing parameter is still measured most accurately by the Super-K atmospheric neutrino experiment. According to the global fit [9], $\theta_{23}=45 \pm 4$ deg. and $\Delta m_{23}^{2}=2.40_{-0.11}^{+0.12} \times 10^{-3} \mathrm{eV}^{2}$ at $1 \sigma$ has been obtained. The parameters are also determined rather accurately.

4. $\theta_{13}$

There have been no single experiment that showed evidence for non-zero $\theta_{13}$. Only the upper 
limit is known; $\sin ^{2} 2 \theta_{13}<0.15$ [15]. However, it might be possible to get some hint for nonzero $\theta_{13}$ by an analysis that combine many experimental results (global analysis). The data used in these analyses are from solar, atmospheric, reactor and accelerator long baseline experiments. In Ref. [16], it was pointed out that an global analysis based on the most updated data indicated non-zero $\theta_{13}$ at $90 \%$ C.L. The indication comes from; (a) a slight disagreement in the best fit $\theta_{12}$ from solar and KamLAND experiments in the 2 flavor framework, and (b) atmospheric neutrino data combined with accelerator and $\mathrm{CHOOZ}$ data. This is an interesting hint. However, the result still depends on the details of the analysis. Indeed, in Ref. [9], the analysis result showed less significant indication for non-zero $\theta_{13}$ (slightly less than $1 \sigma$ effect). The situation is not clear yet. However, these analyses indicate the importance for improving the accuracy of the data further.

\section{Prospects for future solar neutrino experiments}

Solar neutrino problem has been solved essentially by the MSW-LMA solution. Probably, the main goals for future solar neutrino experiments should be studies of the Sun knowing the nature of neutrinos, further confirmation of the MSW-LMA solution and searches for new physics beyond the standard MSW oscillation.

Currently, Super-K is trying to lower the analysis threshold for the solar neutrino measurement. Super-K has been improving the radio purity in the detector water. Recent data have shown that the background level at the upper-central region of the fiducial volume has been reduced by about a factor of 3 relative to the Super-K-I purity [12]. It is likely that Super-K can lower the analysis threshold below $5 \mathrm{MeV}$. In SNO, there is an activity to lower the analysis threshold below $5 \mathrm{MeV}$ [17]. The study suggests that this should be possible. However, a good understanding of the radio purity is required. These studies in Super-K and SNO could lead to the observation of the predicted up-turn of the oscillation probability in the low energy part of the ${ }^{8} \mathrm{~B}$ spectrum.

${ }^{7} \mathrm{Be}$ solar neutrinos have been measured by Borexino. The next major goals in this energy region should be the detection of $C N O$ and pep solar neutrinos, the measurement of $C N O$ neutrinos should give important information on the interim of the Sun. The flux of pep neutrinos have been predicted accurately (2\%). Therefore, a precise measurement of the flux of pep neutrinos should give important information on the oscillation parameters.

In addition to Borexino, KamLAND [18] and SNO+ [19] are trying to measure these neutrinos. KamLAND is purifying the liquid scintillator in the detector. The purification activity in 2007 showed that it is possible to purify the scintillator substantially. The purification has been continuing in 2008. SNO+ is likely to start the experiment with the double beta decay mode. However, the measurement of the ${ }^{7} \mathrm{Be}$, pep and $C N O$ solar neutrinos is also a serious option. Finally we note that there are many activities that try to measure $p p$ solar neutrinos (see, for example [17]).

\section{Prospects for future atmospheric neutrino experiments}

Atmospheric neutrino experiments have been contributing to the oscillation studies related to $\theta_{23}$ and $\Delta m_{23}^{2}$. At present there are many large scale activities to study neutrino oscillations in more detail using accelerator beams. It is likely that these future accelerator experiments will take over studies that have been carried out by atmospheric neutrino experiments. Probably, future 
atmospheric neutrino experiments should try to carry out unique measurements that are relatively difficult by accelerator experiments.

The neutrino flight length ranges up to $12800 \mathrm{~km}$ in atmospheric neutrino experiments. Therefore a large matter resonance is expected to occur for neutrinos (anti-neutrinos) in the energy range of 5 to $10 \mathrm{GeV}$, if the mass hierarchy is normal (inverted). There have been studies carried out for a large water Cherenkov detector [20,21] and for a large iron calorimeter experiment (INO) [22]. In a water Cherenkov experiment, a resonant electron appearance signal can mainly be measured, while in an iron calorimeter experiment, a change in the muon disappearance probability at the resonance energy region can be measured. In a magnetized iron calorimeter experiment, the charge of the muon is measured. Therefore, it is possible to discriminate the mass hierarchy. On the other hand, in a water Cherenkov detector, it is possible to know if the resonant appearance is due to $v_{e}$ or $\bar{v}_{e}$ by studying the $\mathrm{d} \sigma / \mathrm{d} y$ distribution, where $y$ is $\left(E_{v}-E_{\text {lepton }}\right) / E_{v}$. Detailed studies showed that if the total exposure exceeds approximately $1 \mathrm{Mton} \cdot \mathrm{yr}$ and if $\sin ^{2} 2 \theta_{13}$ is larger than about 0.05 , it is possible to determine the mass hierarchy [20,21, 22].

So far, we have neglected the oscillation terms that are related to solar neutrinos $\left(\theta_{12}\right.$ and $\left.\Delta m_{12}^{2}\right)$. The oscillation length relevant to $\Delta m_{12}^{2}$ is shorter than the diameter of the earth for $E_{v}$ below $1 \mathrm{GeV}$. Therefore the effects due to the solar oscillation terms can be visible in atmospheric neutrino experiments. It has been pointed out that these terms could play unique roles in the atmospheric neutrino oscillations, such as the possible measurement of $\sin ^{2} \theta_{23}$, (i.e., the discrimination of the octant of $\theta_{23}$ ) [23]. For simplicity, we assume that $\theta_{13}=0$. The change in the atmospheric $v_{e}$ flux due to oscillations driven by the solar oscillation terms is written as [23];

$$
\frac{F_{v e}^{o s c}}{F_{v e}^{0}}-1=P_{2}\left(r \cos ^{2} \theta_{23}-1\right),
$$

where $F_{v e}^{o s c}$ and $F_{v e}^{0}$ are the atmospheric $v_{e}$ fluxes with and without oscillations, $r\left(\equiv F_{v \mu}^{0} / F_{v e}^{0}\right)$ is the ratio of the un-oscillated atmospheric $v_{\mu}$ and $v_{e}$ fluxes, and $P_{2}$ is the two neutrino transition probability $\left(v_{e} \rightarrow v_{x}\right)$ in matter driven by the solar oscillation terms. $P_{2}$ is large for neutrinos passing through the earth with the energies below $1 \mathrm{GeV}$. Thus the sub-GeV atmospheric neutrinos play an important role in observing the solar term effect. Since the $v_{\mu}$ and $v_{e}$ flux ratio $(r)$ is approximately 2 in the sub-GeV neutrino energy region, the $F_{v e}^{o s c} / F_{v e}^{0}$ value in Eq.6.1 is very close to 1 in the case of the maximal 2-3 mixing (i.e., $\theta_{23}=45^{\circ}$ ). However, according to Eq.6.1, if $\theta_{23}$ is in the first (second) octant $\left(\theta_{23}<45^{\circ}\left(>45^{\circ}\right)\right)$, an excess (deficit) of the sub-GeV $e$-like events is expected. Future atmospheric neutrino experiments should have a unique possibility of measuring the octant of $\theta_{23}$ [24].

\section{Summary}

The studies of the atmospheric and solar neutrinos have been contributing significantly to the understanding of the neutrino masses and mixing angles. $\left|\Delta m_{23}^{2}\right|, \sin ^{2} 2 \theta_{23} \Delta m_{12}^{2}$ and $\sin ^{2} \theta_{12}$ are already measured accurately. Future long baseline experiments are likely to take over some of the topics so far studied by these experiments. However, we believe that future solar and atmospheric neutrino experiments can still get unique information of the properties of neutrinos. 
We thank the organizers of this conference for the kind invitation. This work is partly supported by the Grant-in-Aid in Scientific Research of JSPS.

\section{References}

[1] Y. Fukuda, et al., Phys. Rev. Lett. 81 (1998) 1562.

[2] B. Aharmim, et al., Phys. Rev. Lett. 101 (2008) 111301.

[3] B. Aharmim et al., Phys. Rev. C 72 (2005) 055502.

[4] S. Abe et al., Phys. Rev. Lett. 100 (2008) 221803.

[5] A. Friedland, C. Lunardini, and C. Pena-Garay, Phys. Lett. B 594, (2004) 347.

[6] O.G. Miranda, M.A. Tortola, and J.W.F. Valle, JHEP 10, (2006) 008.

[7] C. Arpesella et al., Phys. Lett. B 658, (2008) 101.

[8] C. Arpesella et al., Phys. Rev. Lett. 101, (2008) 091302.

[9] T. Schwetz, M. Tortola and J.W.F. Valle, arXiv:0808.2016 [hep-ph].

[10] Y. Ashie et al., Phys. Rev. D 71, 112005 (2005).

[11] M. Honda et al., Phys. Rev. D 75, (2007) 043006.

[12] J. Raaf (for the Super-K collaboration), talk presented at the XXIII International Conference on Neutrino Physics and Astrophysics (Neutrino 2008), Christchurch, New Zealand, May 2008.

[13] P. Adamson, et al. (MINOS collaboration), Phys. Rev. Lett. 101 (2008) 131802; N. Saoulidou, Status and prospects for long baseline experiments, in proceedings of 10th International Workshop on Neutrino Factories, Super beams and Beta beams (NuFact08), June 30 - July 5 2008, Valencia, Spain POS (NUFACT08) 003.

[14] M.H. Ahn et al., Phys. Rev. D 74, (2006) 072003.

[15] M. Apollonio et al., Eur. Phys. J. C27, (2003) 331.

[16] G.L. Fogli et al., Phys. Rev. Lett. 101 (2008) 141801.

[17] J. Klein, talk presented at the XXIII International Conference on Neutrino Physics and Astrophysics (Neutrino 2008), Christchurch, New Zealand, May 2008.

[18] P. Decowski, talk presented at the XXIII International Conference on Neutrino Physics and Astrophysics (Neutrino 2008), Christchurch, New Zealand, May 2008.

[19] M.C. Chen (for the SNO+ collaboration), arXiv:0810.3694 [hep-ex].

[20] T. Kajita et al., Proceedings of the 5th Workshop on Neutrino Oscillations and their Origin (NOON2004) Feb. 2004, Tokyo, Japan, p.73.

[21] M. Shiozawa, et al., Proceedings of the RCCN International Workshop on Sub-dominant Oscillation Effects in Atmospheric Neutrino Oscillations, Dec. 2004, Kashiwa, Japan, p.57.

[22] M. Sajiad Athar et al., India-based Neutrino Observatory, project report, INO/2006/01 (2006).

[23] O.L.G. Peres and Y.Au. Smirnov, Nucl. Phys. B 680 (2004) 479.

[24] S. Nakayama (for the Super-Kamiokande collaboration), Proceedings of the RCCN International Workshop on Sub-dominant Oscillation Effects in Atmospheric Neutrino Oscillations, Dec. 2004, Kashiwa, Japan, p.43. 